\title{
BMJ
}

\section{Community based lifestyle intervention for blood pressure reduction in children and young adults in developing country: cluster randomised controlled trial}

\author{
Tazeen $\mathrm{H}$ Jafar, professor of community health sciences and medicine (nephrology), ${ }^{1}$ Muhammad Islam, \\ senior instructor in community health sciences (statistician), ${ }^{1}$ Juanita Hatcher, professor of community health \\ sciences (statistician) , 'Shiraz Hashmi, project coordinator , 'Rasool Bux, data manager, 'Ayesha Khan, \\ nutritionist, ${ }^{1}$ Neil Poulter, professor of preventive cardiovascular medicine, ${ }^{2}$ Salma Badruddin, professor of \\ community health sciences (nutritionist) , ${ }^{1}$ Nish Chaturvedi, professor of clinical epidemiology, ${ }^{2}$ for the \\ Hypertension Research Group
}

\footnotetext{
'Departments of Medicine and Community Health Sciences, Aga Khan University, Karachi, Pakistan

${ }^{2}$ National Heart and Lung Institute, Imperial College London, London Correspondence to: $\mathrm{T} \mathrm{H}$ Jafar tazeen.jafar@aku.edu

Cite this as: $B M J$ 2010;340:c2641 doi:10.1136/bmi.c2641
} based lifestyle intervention on blood pressure in children

\section{ABSTRACT}

Objective To assess the effectiveness of a community and young adults in a developing country setting.

Design Cluster randomised controlled trial.

Setting 12 randomly selected geographical census based clusters in Karachi, Pakistan.

Participants 4023 people aged 5-39 years. Intervention Three monthly family based home health education delivered by lay health workers.

Main outcome measure Change in blood pressure from randomisation to end of follow-up at 2 years.

Results Analysed using the intention to treat principle, the change in systolic blood pressure (adjusted for age, sex, and baseline blood pressure) was significant; it increased by 1.5 (95\% confidence interval 1.1 to 1.9$) \mathrm{mm} \mathrm{Hg}$ in the control group and by 0.1 (-0.3 to 0.5$) \mathrm{mm} \mathrm{Hg}$ in the home health education group ( $\mathrm{P}$ for difference between groups $=0.02$ ). Findings for diastolic blood pressure were similar; the change was $1.5 \mathrm{~mm} \mathrm{Hg}$ greater in the control group than in the intervention group $(\mathrm{P}=0.002)$.

Conclusions Simple, family based home health education delivered by trained lay health workers significantly ameliorated the usual increase in blood pressure with age in children and young adults in the general population of Pakistan, a low income developing country. This strategy is potentially feasible for up-scaling within the existing healthcare systems of Indo-Asia.

Trial registration Clinical trials NCT00327574.

\section{INTRODUCTION}

Cardiovascular disease is now the leading cause of premature mortality worldwide, both in developed countries and in many developing countries. ${ }^{12}$ Hypertension confers the highest attributable risk to deaths from cardiovascular disease. ${ }^{1}$ Epidemiological data provide convincing evidence that the risk of cardiovascular disease related to blood pressure is graded and continuous. ${ }^{3}$ This risk is evident even in childhood, with elevated blood pressure predicting hypertension in adulthood, ${ }^{4}$ and adverse effects of elevated blood pressure in childhood on vascular structure and function, specifically left ventricular hypertrophy, are already apparent in youth. ${ }^{5-7}$ Reduction of blood pressure reduces this risk in people with and without hypertension and is a desired goal in children and adults. ${ }^{489}$

From a public health perspective, shifting the distribution curve of blood pressure by a small amount in an entire population can have a substantial effect on death rates, the overall magnitude of benefit of which is likely to exceed that resulting from treating only people with hypertension. ${ }^{10}$ Strategies to achieve even modest lowering of population levels of blood pressure in children and young adults are therefore important public health goals. Unfortunately, evidence from developed countries suggests that community based strategies for lowering blood pressure are unlikely to yield promising results. ${ }^{11}$ However, reports of health promotion activities in developing countries on blood pressure control are scarce.

Previously, we have shown that children in Pakistan have higher body mass index adjusted blood pressure levels than do white children in the United States. ${ }^{12}$ The burden of hypertension in adults is also high; it affects one in three adults aged 40 years and above, ${ }^{13}$ which is similar to estimates from neighbouring Indian-Asian countries. ${ }^{14}$ The healthcare infrastructure in this region is grossly dysfunctional, especially as regards management of hypertension and associated chronic non-communicable diseases. ${ }^{15}$ Effective population based strategies for blood pressure reduction are thus highly desirable.

We did a cluster randomised controlled trial in Karachi, Pakistan, to assess the impact on blood pressure of a strategy based on community home health education. In addition, in this trial, adults aged 40 and above with hypertension were further randomised, in a factorial design, to trained versus untrained general 


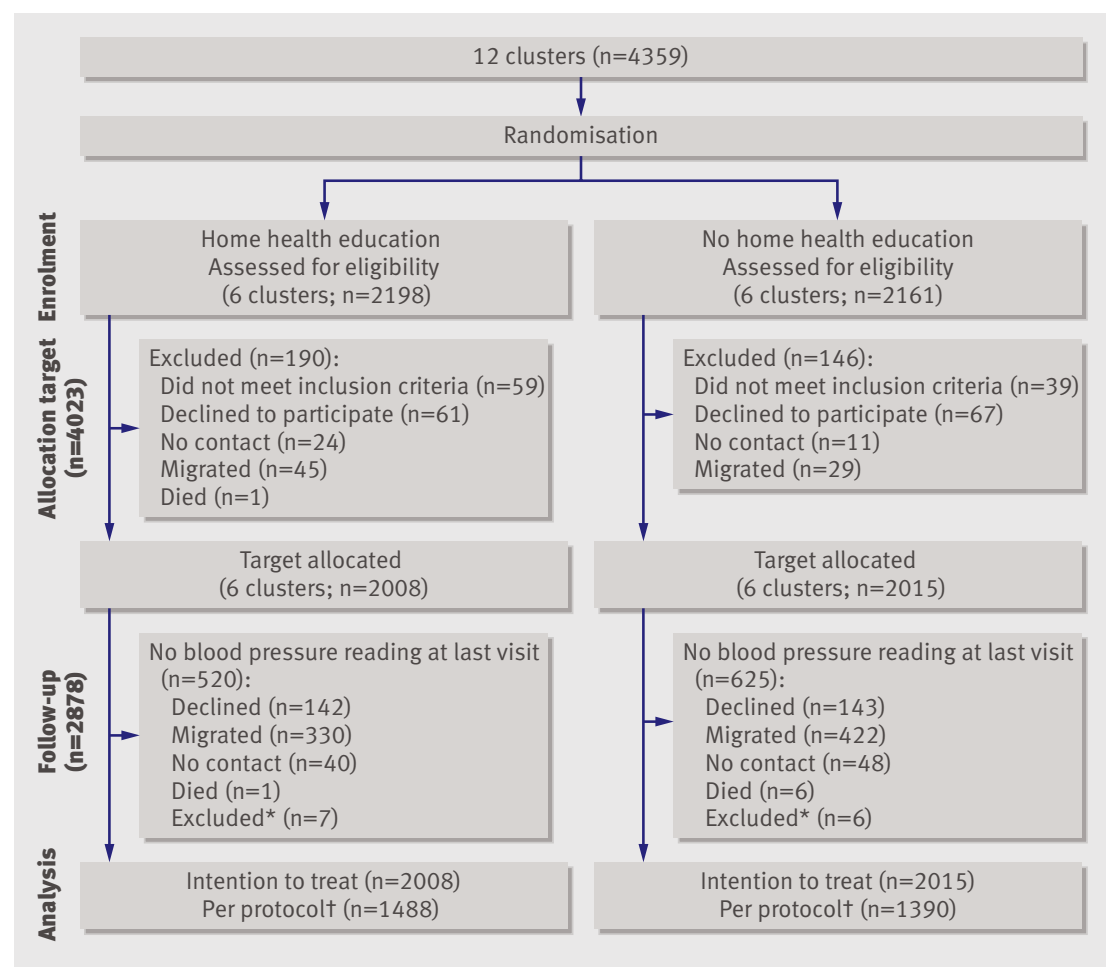

Flow diagram of study. *Participants excluded for protocol violation. †Participants who successfully completed two years of follow-up and were included in analyses

practice management of hypertension. The findings of this factorial trial in adults have been published as a separate report. ${ }^{16}$ Here we present the results in people aged between 5 and 39 years. We hypothesised that children and young adults residing in clusters randomised to home health education would have a beneficial reduction in blood pressure levels compared with controls.

\section{METHODS}

\section{Study design and setting}

This was a cluster randomised controlled trial designed to determine the impact of family based home health education on blood pressure in children and adults at a community level. The rationale for a cluster approach was based on our objective to assess the effectiveness of home health education as a health systems intervention at a population level. The study was started in 2004 and ended in 2007.

\section{Sampling frame and randomisation}

The sampling frame has been described previously. ${ }^{16}$ In brief, using a multistage random sampling technique, we selected 12 out of 4200 low to middle income (mean household monthly income \$70 (£49; €56)) clusters based on a geographical census (about 250 households listed in each cluster) in Karachi, the most populous city in Pakistan, with 16 million inhabitants. Each selected cluster had to be at least $10 \mathrm{~km}$ away from any other selected cluster to minimise the risk of contamination. If this was not the case, we discarded that cluster and selected a new one.
We used computer generated codes to randomly assign six clusters each to home health education and no home health education. The data manager used SPSS software to do this.

\section{Participants, screening, and recruitment}

Field teams masked to randomisation status invited households in the 12 selected clusters to participate. Adults, children, and their parents gave informed consent. In consenting households, one adult aged 15-39 years and one child aged 5-14 years were randomly selected for clinical assessment. We excluded pregnant women, people unable to give informed consent, and patients confined to bed.

Trained field workers masked to the randomisation status of clusters visited the households to collect baseline data. Weight was recorded to the nearest $0.1 \mathrm{~kg}$ (Tanita Solar Powered Digital Scale model 1631) and height to the nearest $0.5 \mathrm{~cm}$ (portable Stadiometer). Body mass index was calculated as weight in kilograms divided by height in meters squared. Resting brachial blood pressure was measured with a calibrated automated device (Omron HEM-737 Intellisense Blood Pressure Monitor) in the sitting position after five minutes of rest with an appropriately sized paediatric or adult cuff, as applicable. ${ }^{17}$ Three consecutive readings were obtained five minutes apart, and we used the last two in the analysis. Research staff were rigorously trained in standardised measurement techniques, with re-training at frequent intervals throughout the study.

\section{Intervention}

\section{Home health education}

Six community health workers, who usually have around eight to 10 years of schooling, were trained in methods of conveying standardised health education messages, using behaviour change communication strategies, to all households in clusters assigned to home health education. One community health worker was assigned to each cluster. The training took place over six weeks according to a case based curriculum on nutrition and healthy lifestyles developed by a team consisting of a nutritionist, a dietitian, and clinicians. Health messages included information on the deleterious effects of hypertension and nondrug interventions for preventing and controlling hypertension and cardiovascular disease. Advice was given on diet and the importance of engaging in moderate physical activity, maintaining normal body weight, and tobacco cessation. The nutritional recommendations were modelled on the dietary approaches to stop hypertension (DASH) diet, adapted to the dietary patterns of the study population. ${ }^{18}$ The dietary advice focused on reducing salt intake; consuming a diet rich in fruit, vegetables, and low fat dairy products; and reducing intake of total fat, especially saturated and hydrogenated fat. Suggestions were given for modifying traditional recipes to reduce fat content, retaining cultural acceptability and economic feasibility. This advice was modified for children to ensure 
Table 1|Characteristics of participants aged 5-39 years by randomisation status

\begin{tabular}{|c|c|c|}
\hline Characteristics & $\begin{array}{l}\text { Home health education } \\
\qquad(\mathrm{n}=2008)\end{array}$ & $\begin{array}{l}\text { No home health education } \\
\qquad(n=2015)\end{array}$ \\
\hline \multicolumn{3}{|l|}{ Cluster } \\
\hline No of clusters & 6 & 6 \\
\hline No of households & 1329 & 1321 \\
\hline Mean (SD) No of residents per household & $6.6(3.3)$ & $6.5(3.0)$ \\
\hline Illiteracy in adults aged $15-39$ years (\%) & 11.5 & 13.8 \\
\hline \multicolumn{3}{|l|}{ Participants } \\
\hline Mean (SD) age (years) & $18.9(9.8)$ & $19.0(9.8)$ \\
\hline No (\%) male & $929(46.3)$ & $923(45.8)$ \\
\hline Mean (SD) body mass index $\left(\mathrm{kg} / \mathrm{m}^{2}\right)$ & $19.8(5.9)$ & $19.8(5.8)$ \\
\hline Mean (SD) systolic blood pressure $(\mathrm{mm} \mathrm{Hg})$ & $114(14)$ & $115(14)$ \\
\hline Mean (SD) diastolic blood pressure $(\mathrm{mm} \mathrm{Hg})$ & $74(11)$ & $74(11)$ \\
\hline No (\%) current tobacco use for age $15-39$ years* & $286 / 1173(24.4)$ & $252 / 1175(21.4)$ \\
\hline
\end{tabular}

adequate calorie and nutrient intake for optimum growth. ${ }^{19}$

The first home health education session, lasting 90 minutes, was held at a time when all members of the household were present. Follow-up reinforcement visits of 30 minutes took place at three monthly intervals.

\section{Follow-up procedure}

Trained outcomes assessors masked to randomisation status evaluated all participants two years after randomisation. The outcomes assessors were not part of and had no relationship with the baseline data collection or the community health worker team. Three consecutive blood pressure readings were taken, according to the same protocol used at baseline. Home visits were made during the first half of the day and balanced between randomised clusters to minimise variability due to diurnal and seasonal variation in blood pressure. ${ }^{20}$

\section{Outcomes and measurements}

The primary outcome was change in systolic blood pressure from baseline to the final follow-up visit at two years. The secondary outcome was change in diastolic blood pressure from baseline to the final visit. Tertiary outcomes included change in body mass index and current tobacco use (smoked or chewable) status.

\section{Statistical analysis}

We calculated our planned sample size of 2100 participants in each arm of the trial (total of 4200) on the assumption of six clusters per group, a dropout rate of $20 \%$, power of $90 \%$, and $\alpha=0.05$ to detect a difference in change in systolic blood pressure of 1.5 (SD 11) mm $\mathrm{Hg}$ between the home health education and no home health education groups. ${ }^{21}$ We assumed an intraclass correlation of 0.001 on the basis of previous experience. $^{1622}$

We used SAS version 9.13 for statistical analyses. For the main intention to treat analysis, we assigned cluster specific mean blood pressure values of participants who completed two years of follow-up $(\mathrm{n}=2878)$ to those with missing blood pressure readings at the final visit ( $\mathrm{n}=1145)$. This method of imputation of missing values has been shown to yield valid inferences and is recommended for large community intervention trials with small cluster sizes. ${ }^{23}$ We accounted for clustering at the level of census by household as a random effect in all analyses, as this was also the unit of randomisation. ${ }^{24}$ As the community health workers delivering home health education were assigned to one intervention cluster each, this strategy also accounted for clustering by community health worker. We compared the primary and secondary outcomes between randomised clusters by using nested analysis of variance with a mixed factorial design, accounting for clustering. We considered a $\mathrm{P}$ value of less than 0.05 to be statistically significant for the main effects. We report means and 95\% confidence intervals for the treatment effects, adjusted for sex, age, and baseline blood pressure in the model.

We also did sensitivity analyses on our findings as per protocol, after excluding participants with missing readings at the last follow-up visit and after replacing missing readings with the mean follow-up blood pressure of all 12 clusters combined. In addition, we did subgroup analyses for the primary and secondary outcomes after stratification by age groups by using the main intention to treat approach. For the tertiary outcomes, we used generalised estimation equation analysis for changes in body mass index and mixed model analysis of variance for current tobacco use.

\section{RESULTS}

We randomly assigned 12 clusters with 2650 households and 4023 people aged 5-39 years - six clusters to each of the study groups. The figure shows the trial profile.

\section{Baseline characteristics}

The mean age of participants - about half of whom were male - was 18.9 years (table 1). The mean systolic blood pressure was 114 (SD 14) $\mathrm{mm} \mathrm{Hg}$, and the mean diastolic blood pressure was 74 (11) $\mathrm{mm} \mathrm{Hg}$.

\section{Missing data}

During follow-up, blood pressure readings were not available at two years for $1145(28 \%)$ participants (figure). Thus, $2878(71.5 \%)$ participants-1488 (74\%) in the home health education group and $1390(69 \%)$ in the no home health education group-successfully completed two years of follow-up.

The distribution of participants with missing versus non-missing final blood pressure readings did not differ significantly by group allocation in terms of baseline systolic blood pressure (115 (15) v 114 (14) $\mathrm{mm} \mathrm{Hg}$, $\mathrm{P}=0.6)$ or diastolic blood pressure $(74$ (11) v74 (11) mm $\mathrm{Hg}, \mathrm{P}=0.5)$.

Primary and secondary outcomes measures: systolic and diastolic blood pressure

Final mean systolic and diastolic blood pressure levels were significantly greater in the control group (116/ 
Table 2 Change in blood pressure (95\% confidence interval) from baseline to follow-up by intervention allocation $(n=4023)$

\begin{tabular}{lccc} 
Blood pressure & $\begin{array}{c}\text { Home health education } \\
(\mathrm{n}=2008)\end{array}$ & $\begin{array}{c}\text { No home health education } \\
(\mathrm{n}=2015)\end{array}$ & P value \\
Systolic $(\mathrm{mm} \mathrm{Hg})^{*}$ & $0.1(-0.3$ to 0.5$)$ & $1.5(1.1$ to 1.9$)$ & 0.02 \\
\hline Diastolic $(\mathrm{mm} \mathrm{Hg}) \dagger$ & $0.6(0.3$ to 0.9$)$ & $2.1(1.8$ to 2.4$)$ & 0.002 \\
\hline
\end{tabular}

*Adjusted for clustering, age, sex, and baseline systolic blood pressure.

†Adjusted for clustering, age, sex, and baseline diastolic blood pressure.

$76 \mathrm{~mm} \mathrm{Hg}$ ) than in the home health education group $(114 / 74 \mathrm{~mm} \mathrm{Hg})(\mathrm{P}=0.02 / 0.005)$. The intraclass correlation for systolic blood pressure was 0.008 .

Systolic blood pressure (adjusted for age, sex, and baseline pressure) increased significantly from baseline to final visit in the control group by 1.5 (95\% confidence interval 1.1 to 1.9$) \mathrm{mm} \mathrm{Hg}$. In contrast, systolic blood pressure did not change appreciably over the course of the trial $(0.1(-0.3$ to 0.5$) \mathrm{mm} \mathrm{Hg})$ in the home health education group. The difference in change in systolic blood pressure between the groups was statistically significant $(\mathrm{P}=0.02)$ (table 2). Unlike systolic blood pressure, diastolic pressure increased significantly in both randomised groups, but this rise was significantly higher in the control group $(\mathrm{P}=0.002$ for difference between groups) (table 2). The results of the sensitivity analyses were in the same direction as the main findings (table 3).

The subgroup analyses stratified by age also yielded consistent results. In children aged 5-14 years, the change in systolic blood pressure adjusted for age, sex, and baseline blood pressure was 1.8 (1.2 to 2.4) $\mathrm{mm} \mathrm{Hg}$ in the home health education group compared with 2.9 (2.3 to 3.5$) \mathrm{mm} \mathrm{Hg}$ in the no home health education group $(\mathrm{P}=0.13)$. The corresponding changes in diastolic blood pressure were 1.3 (0.8 to 1.8) and 2.6 (2.1 to 3.1$) \mathrm{mm} \mathrm{Hg}(\mathrm{P}=0.06)$. In adults aged $15-39$, the change in systolic blood pressure adjusted for age, sex, and baseline pressure was $-1.1(-1.7$ to -0.6$) \mathrm{mm} \mathrm{Hg}$ in the intervention group and 0.6 (0.1 to 1.2$) \mathrm{mm} \mathrm{Hg}$ in the control group $(\mathrm{P}<0.001)$, and changes in diastolic blood pressure were 0.04 ( -0.38 to 0.46$)$ and 1.84 (1.42 to 2.27$) \mathrm{mm} \mathrm{Hg}(\mathrm{P}<0.001)$.

\section{Tertiary outcome measures}

The proportion of current tobacco users among participants aged 15-39 years decreased from baseline to the final visit in both groups (by $8.5 \%$ in the intervention group and $6.1 \%$ in the control group). The odds ratio for change in current tobacco use between home health education and control was 1.15 (95\% confidence interval 0.95 to 1.40$)(\mathrm{P}=0.16)$. An increase in mean body mass index occurred in both groups in the overall population. The difference between the home health education and control groups was $0.02(-0.29$ to 0.33) $(\mathrm{P}=0.89)$.

\section{DISCUSSION}

Our community based intervention of family based home health education, delivered by a community health worker, had a significant beneficial effect on blood pressure in children and young adults in the developing country setting of Karachi, Pakistan. Over two years, home health education resulted in a blood pressure that was $1.6 / 1.4 \mathrm{~mm} \mathrm{Hg}$ lower in the intervention group than in the control group. Our findings have considerable global public health relevance, as even a modest shift in mean blood pressure at a population level has been shown to have a marked beneficial effect on the primary prevention of stroke and cardiovascular disease. ${ }^{25}$

\section{Comparison with other studies}

Previous studies of the effect of health promotion on blood pressure, such as the North Karelia Study and the Pawtucket Heart Study, have yielded inconclusive results. A similar effort to reduce dietary salt in subSaharan Africa tended to lower blood pressure levels in the short term. ${ }^{26}$ However, many of these studies had deficiencies in study design and considerable contamination bias, and some have been demonstration projects without a concomitant control group. ${ }^{68101127-30}$

A recent Cochrane collaboration concluded that community health worker programmes of healthcare delivery were of significant benefit in areas such as malaria treatment, immunisation, infant mortality, and breast feeding. ${ }^{3132}$ Such models used to be the dominant mode of healthcare delivery in China for people with poor access to effective care and are being proposed again to re-establish universal health coverage. ${ }^{33-35}$ The government funded lady health workers programme of Pakistan has been implemented for about two decades and provides immunisation, basic maternal, and preventive child care services. ${ }^{31}$

Models of service by lay health workers are operational in several under-resourced countries including India, Kenya, Uganda, Ghana, Ethiopia, South Africa, and China. Our results show that a similar vehicle can be used for health promotion with effective lowering of blood pressure levels in the population.

The impact of health promotion on blood pressure in our study could have been mainly due to the poor educational attainment and health literacy of our study population, thus leaving considerable scope for improvement in behaviour with sound communication strategies. However, these attributes are characteristic of many developing countries, to which our findings may be generalisable. ${ }^{3637}$

\section{Limitations and strengths}

The main limitation of the trial was that its duration was short, so that we cannot tell to what extent changes in blood pressure can be sustained; nor can we speculate on the post-trial impact of the intervention, which has been variable in other trials. ${ }^{3839}$ A medium term (four year) post-trial follow-up of blood pressure levels is planned. Furthermore, our trial was not sufficiently long or large to determine the effect of our interventions on downstream cardiovascular outcomes. Such a trial would clearly be expensive and take several years to complete. Convincing funders to support 
Table $3 \mid$ Change in blood pressure (95\% confidence interval) from baseline to follow-up by intervention allocation: sensitivity analyses

\begin{tabular}{|c|c|c|c|}
\hline Sensitivity analyses & Home health education & No home health education & $P$ value \\
\hline Per protocol approach & $(n=1488)$ & $(n=1390)$ & \\
\hline Mean systolic blood pressure at follow-up (mm Hg)* & 113.8 (113.1 to 114.5$)$ & 115.5 (114.8 to 116.2$)$ & 0.06 \\
\hline Mean diastolic blood pressure at follow-up $(\mathrm{mm} \mathrm{Hg})^{\star}$ & 74.2 (73.7 to 74.7$)$ & $76.0(75.4$ to 76.5$)$ & 0.04 \\
\hline Change in systolic blood pressure $(\mathrm{mm} \mathrm{Hg}) \dagger$ & $0.4(-0.2$ to 0.9$)$ & $1.5(1.0$ to 2.1$)$ & 0.10 \\
\hline Change in diastolic blood pressure $(\mathrm{mm} \mathrm{Hg}) \ddagger$ & $0.8(0.4$ to 1.3$)$ & 2.1 (1.7 to 2.5$)$ & 0.06 \\
\hline $\begin{array}{l}\text { Replacing missing final values with overall mean follow-up } \\
\text { reading for all clusters combined }\end{array}$ & $(n=2088)$ & $(n=2015)$ & \\
\hline Mean systolic blood pressure at follow-up $(\mathrm{mm} \mathrm{Hg})^{*}$ & 114.1 (113.6 to 114.6$)$ & 115.7 (115.2 to 116.2$)$ & 0.07 \\
\hline Mean diastolic blood pressure at follow-up $(\mathrm{mm} \mathrm{Hg})^{\star}$ & 74.4 (74.0 to 74.8$)$ & 75.7 (75.3 to 76.1$)$ & 0.03 \\
\hline Change in systolic blood pressure $(\mathrm{mm} \mathrm{Hg}) \dagger$ & $0.1(-0.3$ to 0.5$)$ & $1.0(0.6$ to 1.5$)$ & 0.06 \\
\hline Change in diastolic blood pressure $(\mathrm{mm} \mathrm{Hg}) \ddagger$ & $0.6(0.3$ to 0.9$)$ & $1.6(1.3$ to 2.0$)$ & 0.01 \\
\hline
\end{tabular}

a trial required demonstration that such an intervention in a developing country setting could indeed influence blood pressure in the first place. Importantly, the atherosclerotic process is well documented as starting during childhood and is established in young adults, indicating that early interventions are needed to slow or reverse the process. ${ }^{4041}$ Moreover, even a 1-2 $\mathrm{mm} \mathrm{Hg}$ reduction in systolic blood pressure has been shown to reduce hypertension by $10 \%$ in children. ${ }^{4}$ In addition, the benefit of a modest reduction in systolic blood pressure at a population level has been shown to translate into substantial reductions in deaths from stroke and coronary heart disease; each $2 \mathrm{~mm} \mathrm{Hg}$ reduction in systolic blood pressure reduces deaths from stroke by $10 \% .{ }^{42}{ }^{43}$ Sound evidence thus supports a direct link between blood pressure reduction in youth and protection from hypertension and hard cardiovascular outcomes in the young and older populations.

An additional limitation potentially affecting the generalisability of this trial was that final blood pressure measurements were not available on $28 \%$ of the study population. However, baseline blood pressure did not differ between those for whom we did and did not manage to obtain a final blood pressure recording. In addition, our main analysis was based on the intention to treat principle, and results were consistent with the per protocol analysis and in the subgroup analysis stratified by age groups. Therefore, we believe our findings to be robust.

As the control group received no visits for health promotion, we cannot assess which component of home health education was beneficial. However, answering this question was not the intention of the trial, which was designed to assess home health education as a composite "packaged" approach of various components emphasising adoption of healthy behaviours, including a diet rich in fruit and vegetables and low in salt and fat, weight management, and physical activity. In addition, although the outcomes assessors were masked, knowledge of treatment allocation is always a possibility in a community trial of this nature. However, automated Omron devices were used to measure the outcome of blood pressure, and their digital display of blood pressure is not subject to potential observer bias.

Our study has several strengths. This is the first population based randomised controlled trial of its kind in Indo-Asia to test the impact of such strategies in lowering blood pressure levels in the population. The sampling strategies ensured recruitment of representative clusters. The distance between study clusters $(10 \mathrm{~km})$, lack of evidence of migration among clusters, and visits by health educators only to the clusters randomised to the intervention minimised the chances of cross contamination. The study design ensured that measurements on participants during baseline and follow-up period were obtained simultaneously in home health education and no home health education clusters to account for any potential bias due to seasonal variations in blood pressure. ${ }^{20}$

\section{Policy implications and conclusions}

Although formal economic evaluations of strategies used to reduce the risk of chronic disease will be needed before interventions are scaled up, currently about 100000 female health workers provide basic preventive maternal and child health services to about 80 million people at a per capita cost of $\$ 0.75$, inclusive of human resources, training, and other operative expenses. ${ }^{44}$ The health gains are thus likely to outweigh the nominal marginal cost of adding our modest proposed strategy on to the existing platform of the lady health workers programme in Pakistan.

Our findings provide the first evidence that a family based home health education package delivered by trained lay health workers can lead to an effective reduction in blood pressure in people aged 5-39 years at a community level in a poorly resourced developing country setting. These findings have substantial implications for prevention of hypertension and cardiovascular disease. 


\section{WHAT IS ALREADY KNOWN ON THIS TOPIC}

Even modest reductions in blood pressure at a population level in children and young adults leads to prevention of hypertension and a reduction in cardiovascular morbidity and mortality

However, community based strategies to achieve such shifts in blood pressure have not yielded promising results

\section{WHAT THIS STUDY ADDS}

Home health education delivered by trained lay health workers significantly blunted the usual increase in blood pressure with age in children and young adults in the general population in Pakistan

Such strategies can potentially be adopted by other low income countries for controlling the epidemic of hypertension
12 Jafar TH, Islam M, Poulter N, Hatcher J, Schmid CH, Levey AS, et al. Children in South Asia have higher body mass-adjusted blood pressure levels than white children in the United States: a comparative study. Circulation 2005;111:1291-7.

13 Jafar TH, Levey AS, Jafary FH, White F, Gul A, Rahbar MH, et al. Ethnic subgroup differences in hypertension in Pakistan. J Hypertens 2003;21:905-12.

14 Hypertension Study Group. Prevalence, awareness, treatment and control of hypertension among the elderly in Bangladesh and India: a multicentre study. Bull World Health Organ 2001;79:490-500.

15 Jafar TH, Jessani S, Jafary FH, Ishaq M, Orakzai R, Orakzai S, et al. General practitioners' approach to hypertension in urban Pakistan: disturbing trends in practice. Circulation 2005;111:1278-83.

16 Jafar TH, Hatcher J, Poulter N, Islam M, Hashmi S, Qadri Z, et al. Community-based interventions to promote blood pressure control in a developing country: a cluster randomized trial. Ann Intern Med 2009;151:593-601.

17 O'Brien E, Waeber B, Parati G, Staessen J, Myers MG. Blood pressure measuring devices: recommendations of the European Society of Hypertension. BMJ 2001;322:531-6.

18 Sacks FM, Svetkey LP, Vollmer WM, Appel LJ, Bray GA, Harsha D, et al. Effects on blood pressure of reduced dietary sodium and the Dietary Approaches to Stop Hypertension (DASH) diet. N Engl / Med 2001;344:3-10.

We thank the members of Hypertension Research Group, more than 40 field workers, and support staff (including Rahim Rehmani), as their hard work was critical to the success of the study. Members of the Hypertension Research Group include Fahim Jafary, Aamir Hameed, Ata Khan, Anwar Gilani, Shehla Zaidi, Nadir Syed, Saleem Jessani, Zeeshan Qadri, and Peter Cosgrove. We also thank members of the Data Safety and Monitoring Committee (Umer Daar, Bhojo Khealani, and Yasmin Parpio). We express our appreciation to all participants for their cooperation during the study.

Contributors: $\mathrm{TH}, \mathrm{JH}, \mathrm{NP}$, and NC were responsible for the study concep and design. THJ, JH, SB, AK, SH, and RB were responsible for the implementation of the study and acquisition of data. MI analysed the data with advice from $\mathrm{JH}$ and $\mathrm{TH}$. All authors drafted and critically revised the manuscript for important intellectual content. THJ, JH, NP, and NC obtained funding and are the guarantors.

Funding: The study was financially supported by a research award (070854/Z/03/Z) from the Wellcome Trust, UK. The design and conduct of the study and the analysis, interpretation, and presentation of the data were the responsibility of the authors, with no involvement from the funder.

Competing interests: None declared.

Ethical approval: The Aga Khan University Ethics Review Committee approved the study.

Data sharing: The protocol, training manuals, and statistical code are available from the corresponding author at tazeen.jafar@aku.edu.

1 Lopez AD, Mathers CD, Ezzati M, Jamison DT, Murray CJ. Global and regional burden of disease and risk factors, 2001: systematic analysis of population health data. Lancet 2006;367:1747-57.

2 Phillips M, Feachem RG, Murray CJ, Over M, Kjellstrom T. Adult health: a legitimate concern for developing countries. Am J Public Health 1993;83:1527-30.

3 MacMahon S, Peto R, Cutler J, Collins R, Sorlie P, Neaton J, et al. Blood pressure, stroke, and coronary heart disease: part 1. Prolonged differences in blood pressure: prospective observational studies corrected for the regression dilution bias. Lancet 1990;335:765-74.

4 Ingelfinger JR. Pediatric antecedents of adult cardiovascular disease -awareness and intervention. N Engl/ Med 2004:350:2123-6.

5 Daniels SD, Meyer RA, Loggie JM. Determinants of cardiac involvement in children and adolescents with essential hypertension. Circulation 1990;82:1243-8.

6 Hanevold C, Waller J, Daniels S, Portman R, Sorof J. The effects of obesity, gender, and ethnic group on left ventricular hypertrophy and geometry in hypertensive children: a collaborative study of the International Pediatric Hypertension Association. Pediatrics 2004;113:328-33.

7 Whincup PH, Cook DG, Adshead F, Taylor S, Papacosta O, Walker M, et al. Cardiovascular risk factors in British children from towns with widely differing adult cardiovascular mortality. BMJ 1996;313:79-84.

8 Stamler J, Stamler R, Neaton JD. Blood pressure, systolic and diastolic, and cardiovascular risks: US population data. Arch Intern Med 1993;153:598-615.

9 Hypertension Detection and Follow-up Program Cooperative Group. Five-year findings of the hypertension detection and follow-up program: I. Reduction in mortality of persons with high blood pressure, including mild hypertension. JAMA 1979;242:2562-71.

10 Rose G. Sick individuals and sick populations: 1985. Bull World Health Organ 2001;79:990-6.

11 Ebrahim S, Smith GD. Exporting failure? Coronary heart disease and stroke in developing countries. Int J Epidemiol 2001;30:201-5.
19 Williams CL, Hayman LL, Daniels SR, Robinson TN, Steinberger J, Paridon S, et al. Cardiovascular health in childhood: a statement for health professionals from the Committee on Atherosclerosis, Cardiovascular Disease in the Young, American Heart Association. Circulation 2002;106:143-60.

20 Woodhouse PR, Khaw KT, Plummer M. Seasonal variation of blood pressure and its relationship to ambient temperature in an elderly population. J Hypertens 1993;11:1267-74.

21 Fortmann SP, Winkleby MA, Flora JA, Haskell WL, Taylor CB. Effect of long-term community health education on blood pressure and hypertension control: the Stanford Five-City Project. Am J Epidemiol 1990;132:629-46.

22 Donner A, Klar N. Statistical considerations in the design and analysis of community intervention trials. J Clin Epidemiol 1996;49:435-9.

23 Taljaard M, Donner A, Klar N. Imputation strategies for missing continuous outcomes in cluster randomized trials. Biom J 2008;50:329-45.

24 Eldridge SM, Ashby D, Feder GS, Rudnicka AR, Ukoumunne OC. Lessons for cluster randomized trials in the twenty-first century: a systematic review of trials in primary care. Clin Trials 2004:1:80-90.

25 Eastern Stroke and Coronary Heart Disease Collaborative Research Group. Blood pressure, cholesterol, and stroke in eastern Asia. Lancet 1998;352:1801-7.

26 Cappuccio FP, Kerry SM, Micah FB, Plange-Rhule J, Eastwood JB. A community programme to reduce salt intake and blood pressure in Ghana [ISRCTN88789643]. BMC Public Health 2006;6:13.

27 Nissinen A, Tuomilehto J, Puska P. Management of hypertension and changes in blood pressure level in patients included in the hypertension register of the North Karelia Project. Scand J Soc Med 1980;8:17-23.

28 Carleton RA, Lasater TM, Assaf AR, Feldman HA, McKinlay S. The Pawtucket Heart Health Program: community changes in Health 1995;85:777-85.

29 Tuomilehto J, Puska P, Nissinen A, Salonen J, Tanskanen A Pietinen $\mathrm{P}$, et al. Community-based prevention of hypertension in North Karelia, Finland. Ann Clin Res 1984;16(suppl 43):18-27S.

30 Farquhar JW, Fortmann SP, Flora JA, Taylor CB, Haskell WL, Williams PT, et al. Effects of communitywide education on cardiovascular disease risk factors: the Stanford Five-City Project. IAMA 1990;264:359-65.

31 Haines A, Sanders D, Lehmann U, Rowe AK, Lawn JE, Jan S, et al. Achieving child survival goals: potential contribution of community health workers. Lancet 2007;369:2121-31.

32 Lewin SA, Dick J, Pond P, Zwarenstein M, Aja G, van Wyk B, et al. Lay health workers in primary and community health care. Cochrane Database Syst Rev 2005;1:CD004015.

33 Stark R. Lay workers in primary health care: victims in the process of social transformation. Soc Sci Med 1985;20:269-75.

34 Hsiao WC. Transformation of health care in China. N Engl J Med 1984;310:932-6.

35 Dummer TJ, Cook IG. Health in China and India: a cross-country comparison in a context of rapid globalisation. Soc Sci Med 2008;67:590-605.

36 Weiss BD. Health literacy: an important issue for communicating health information to patients. Zhonghua Yi Xue Za Zhi (Taipei) 2001;64:603-8.

37 Cappuccio FP, Micah FB, Emmett L, Kerry SM, Antwi S, Martin-Peprah R, et al. Prevalence, detection, management, and Hypertension, and Obesity in the Young (AHOY) of the Council on cardiovascular risk factors and projected disease risk. Am J Public 
control of hypertension in Ashanti, West Africa. Hypertension 2004;43:1017-22.

38 Holman RR, Paul SK, Bethel MA, Matthews DR, Neil HA. 10-year follow-up of intensive glucose control in type 2 diabetes. $N$ Engl J Med 2008;359:1577-89.

39 Mortality after 16 years for participants randomized to the Multiple Risk Factor Intervention Trial. Circulation 1996;94:946-51.

40 Newman WP 3rd, Freedman DS, Voors AW, Gard PD, Srinivasan SR, Cresanta JL, et al. Relation of serum lipoprotein levels and systolic blood pressure to early atherosclerosis: the Bogalusa Heart Study. N Engl J Med 1986;314:138-44.

41 Davis PH, Dawson JD, Riley WA, Lauer RM. Carotid intimal-medial thickness is related to cardiovascular risk factors measured from childhood through middle age: the Muscatine Study. Circulation 2001;104:2815-9.

42 Ford ES, Ajani UA, Croft JB, Critchley JA, Labarthe DR, Kottke TE, et al. Explaining the decrease in US deaths from coronary disease, 19802000. N Engl J Med 2007;356:2388-98.

43 Lewington S, Clarke R, Qizilbash N, Peto R, Collins R. Age-specific relevance of usual blood pressure to vascular mortality: a metaanalysis of individual data for one million adults in 61 prospective studies. Lancet 2002;360:1903-13.

44 Ministry of Health, Government of Pakistan. National programme for family planning and primary health care. 2008. www.phc.gov.pk.

Accepted: 14 March 2010 